\title{
The Inductive Approach to Strand Space*
}

\author{
Yongjian $\mathrm{Li}^{1,2}$ \\ ${ }^{1}$ Key Laboratory of Computer Science \\ 2 The State Key Laboratory of Information Security, Institute of Software, \\ Chinese Academy of Sciences, P.O.Box 8718, Beijing, China \\ lyj238@ios.ac.cn
}

\begin{abstract}
Strand space is a promising technique developed by Guttman et al. from MITRE company, and it provides us an intuitive and clear framework to analyze security protocols, but its mechanics of the proof tend to be quite intricate and not necessarily easy to be formalized. In this paper, we combine the inductive approach with strand space. We introduce an inductive definition for bundles, and it not only provides us a constructive illustration for a bundle, but also introduces an effective and rigorous technique of rule induction to prove properties of bundles. Using this induction principle, we not only prove that a bundle is a casually well-founded graph, but also give a rigorous proof for results of authentication tests. Our result of authentication test extends Guttman's result to a more general case, and its proof is also much easier and clearer. As a trivial case study, we prove authentication properties of Needham-Schroeder-Lowe protocol. Our approach has been mechanized using Isabelle/HOL.
\end{abstract}

\section{Introduction}

Strand space is a promising technique developed by Guttman et al. from MITRE company 1. The most important of all for analysis is carried out on the notion of bundles. A bundle is a casually well-founded set of nodes and arrows of both kinds, which sufficiently formalizes a session of a protocol. In a bundle, it must be insured that a node is included only if all nodes that proceed it are already included. For the strand corresponding to the principal in a given protocol run, we construct all possible bundles containing nodes of the strand. In fact, this set of bundles encodes all possible interactions of the environment with that principal in the run. Reasoning about the protocol takes place on this set of bundles. Typically, for the protocol to be correct, each such bundle must contain one strand of each legitimate principals apparently participating this session, all agreeing on principals, nonces, and session keys. Penetrator strands may also be entangled in a bundle, even in a correct protocol, but they should not prevent legitimate parties from agreeing on the data values, or from maintaining the secrecy of the value chosen. The key to this approach is the fact that a bundle

\footnotetext{
* This work is supported by NSF project of China under Grant No.60173020, 60421001, 60223005.
} 
form a finite, well-founded sets under the relation, and each non-empty subset of the bundle has a $\preceq$-minimal element. A powerful idea, authentication test, is also introduced by Guttman et al 22. This is basically a formalization of the basic challenge-response style primitive that is a building block for many protocols. An agent transmits a so-called test component, and later receives back another term that is in some transformed form of the component, then only a regular principal, not penetrator can have transformed it. In favorable circumstances, it can only be one regular participant, the intended one, who has thereby been authenticated.

Although strand space provides us an intuitive and clear framework to analyze why security protocols are correct, it seemed that the mechanics of the proof tend to be quite intricate and not necessarily easy to be formalized. To our knowledge, no one has ever formalized strand space theory in a theorem prover since the theory was introduced in 1998. Note that almost 7 years has passed, which is not a short time. Special attention should be paid to consider why it is so difficult to formalize strand space. In our opinion, two problems are due to this difficulty. Firstly, as the cornerstone in strand space theory, bundle's definition is not suitable for formal reasoning. It is just a sketchy property description of a graph about a protocol session, that is, a bundle is a casually well-founded graph. However, it does not tell us how this graph is constructed. Secondly, many concepts and proofs are very informal and complicated, and it is far away from being mechanized. One evidence is results about authentication tests. Authentication tests provide a general and powerful idea to prove authentication properties in a wide range of security protocols, and they are easy to apply themselves, but the proofs justifying them are quite complicated and difficult to be formalized. In order to prove these results, Guttman has introduced normal bundles and efficient bundles, which place more restriction on the operations of penetrator, and he has proved two important lemmas: a normal form lemma and an efficient form lemma. Many definitions are involved in his proof, such as graph operations, rising and falling paths, bridges, and it is too tedious to formally define them in a theorem prover, and proofs of the two lemmas are so complicated that it is hard to follow even in paper proof, let alone to formalize them and present formal proofs in theorem provers.

The main contributions of this paper are as following: (1) We introduce an inductive definition for bundles, and it not only provides us a constructive illustration for a bundle, but also introduces an effective and rigorous technique of rule induction to prove properties of bundles. (2) We formalize the semantics to fresh assumption, that is certain data items, such as nonces and session keys, are fresh and never arise in more than one protocol run. We also introduce a notion of complete transforming path. Roughly speaking, a complete transforming path $p$ for a data item $a$ is a path such that record necessary transforming information about $a$. Suppose $a$ uniquely originates in $n$, and $n^{\prime}$ is a node containing $a$ in a bundle $b$, then there is a complete transforming path from $n$ to $n^{\prime}$ through $b$ about $a$. (3) We extend Guttman's authentication results to a more general case, and use results of well-foundedness of bundle and complete path to give an easier 
and clearer proof. (4) We formalized our theory in Isabelle/HOL, and prove authentication results of Needham-Schroeder-Lowe protocol as a trivial case study. The remainder of this paper is organized as follows: Section 2 formalizes preliminary definitions such as messages, agents, and strands, strand spaces. Section 3 introduces the inductive definition of bundle and the corresponding induction principle. Section 4 introduces the notion of complete path and authentication results. Section [5] is related work and conclusion.

\section{Strands and Strand Space}

The notions in this subsection are mainly from Guttman's original definition, and we just formalize them straightforward in Isabelle.

datatype sign=positive ("+"100) |negative (" - "100)

types signed_msg $=$ "sign $\times$ msg"

typedecl sigma

types node=" sigma $\times$ nat"

types strand_space=" sigma $\Rightarrow$ signed_msg list "

consts Sigma_set :: "sigma set" (" $\left.\sum "\right)$ )"

SP :: "strand_space"

attr::"sigma $\Rightarrow$ agent"

constdefs Domain::" node set"

$"$ Domain $==\left\{(\mathrm{s}, \mathrm{i}) . \mathrm{s} \in \sum \wedge \mathrm{i}<\operatorname{length}(\mathrm{SP} \mathrm{s})\right\} "$

constdefs casual1::" (node $\times$ node) set"

$$
" \text { casual } 1==\left\{\begin{array}{l}
(\mathrm{n} 1, \mathrm{n} 2) \cdot \mathrm{n} 1 \in \text { Domain } \wedge \mathrm{n} 2 \in \text { Domain } \wedge \\
\text { node_sign } \mathrm{n} 1=+\wedge \text { node_sign } \mathrm{n} 2=-\wedge \\
\wedge \text { node_term } \mathrm{n} 1=\text { node_term } \mathrm{n} 2 \wedge \text { fst } \mathrm{n} 1 \neq \text { fst } \mathrm{n} 2
\end{array}\right\}
$$

syntax "_casual1":: "node $\Rightarrow$ node $\Rightarrow$ bool" (infix " $\rightarrow$ "100)

translations "n1 $\rightarrow \mathrm{n} 2 "=="(\mathrm{n} 1, \mathrm{n} 2) \in$ casual1"

constdefs casual2::" (node $\times$ node) set"

$$
" \text { casual } 2==\left\{\begin{array}{c}
(\mathrm{n} 1, \mathrm{n} 2) \cdot \mathrm{n} 1 \in \text { Domain } \wedge \mathrm{n} 2 \in \text { Domain } \wedge \\
\wedge(\text { fst } \mathrm{n} 1)=(\text { fst } \mathrm{n} 2) \wedge \text { Suc }(\text { snd } \mathrm{n} 1)=\text { snd } \mathrm{n} 2
\end{array}\right\}
$$

syntax "_casual2"::" node $\Rightarrow$ node $\Rightarrow$ bool" (infix " $\Rightarrow " 50$ )

translations "n1 $\Rightarrow \mathrm{n} 2 "=="(\mathrm{n} 1, \mathrm{n} 2) \in$ casual2"

edge $="$ node $\times$ node"

graph $="$ node set $\times$ edge set"

A signed message such as $(+, m)$ is a pair of a sign and a message. We define an abstract type sigma to define the type of signatures of strands. A node $(s, i)$ is just a pair of a strand signature and an integer index. A strand space is just a trace mapping function which maps a strand signature to a list of signed messages. In our discussion, we usually fix a given strand signature set and strand space, so we define them as arbitrary but fixed consts $\sum$ and $S P$ respectively. Given $\sum$ and $S P$, we are only interested in those nodes which is in the image of $\sum$ under the trace mapping function $S P$, and these nodes are defined by the const Domain. Two kinds of casual relation, denoted by $\rightarrow$ and $\Rightarrow$, are introduced on the nodes in Domain. In the definition of $\rightarrow$, node_term $n$, node_sign $n$ are defined to return $n^{\prime}$ s message term and sign respectively. 


\section{Bundles}

Rather than following the way Guttman defined, we introduce a brand-new definition for bundles. It is totally an inductive definition, as shown as follows. consts bundles ::" graph set"

inductive "bundles" intros

Nil: " $(\varnothing, \varnothing) \in$ bundles"

Add_Positive1:" [| b $\in$ bundles; node_sign n $2=+; \mathrm{n} 2 \in$ Domain; $\mathrm{n} 2 \notin$ fst b; $0<$ snd $\mathrm{n} 2 ; \mathrm{n} 1 \in$ fst $\mathrm{b} ; \mathrm{n} 1 \Rightarrow \mathrm{n} 2 \mid] \Longrightarrow(\{\mathrm{n} 2\} \cup$ fst $\mathrm{b},(\mathrm{n} 1, \mathrm{n} 2) \cup$ snd $\mathrm{b}) \in$ bundles" Add_Positive2:" $[\mid \mathrm{b} \in$ bundles; node_sign n2=+; $\mathrm{n} 2 \notin$ fst b; n2 $\in$ Domain; snd $\mathrm{n} 2=0 \mid] \Longrightarrow(\{\mathrm{n} 2\} \cup$ fst $\mathrm{b}$, snd $\mathrm{b}) \in$ bundles"

Add_negtive1: " $[\mid \mathrm{b} \in$ bundles; node_sign $\mathrm{n} 2=-; \mathrm{n} 2 \notin$ fst $\mathrm{b}$; $\mathrm{n} 1 \rightarrow \mathrm{n} 2 \wedge \mathrm{n} 1 \in$ fst $\mathrm{b} \wedge(\forall \mathrm{n} 3 . \mathrm{n} 3 \in$ fst $\mathrm{b} \longrightarrow(\mathrm{n} 1, \mathrm{n} 3) \notin$ snd $\mathrm{b}) ;$ $0<$ snd $\mathrm{n} 2 ; \mathrm{n} 1^{\prime} \in($ fst $\left.\mathrm{b}) ; \mathrm{n} 1^{\prime} \Rightarrow \mathrm{n} 2 \mid\right]$ $\Longrightarrow\left(\{\mathrm{n} 2\} \cup\right.$ fst $\mathrm{b},\left\{(\mathrm{n} 1, \mathrm{n} 2),\left(\mathrm{n} 1^{\prime}, \mathrm{n} 2\right)\right\} \cup$ snd $\left.\mathrm{b}\right) \in$ bundles"

Add_negtive2: " [l b $\in$ bundles; node_sign n2=-; n2 $\notin$ (fst b); $\mathrm{n} 1 \rightarrow \mathrm{n} 2 \wedge \mathrm{n} 1 \in$ fst $\mathrm{b} \wedge(\forall \mathrm{n} 3 . \mathrm{n} 3 \in$ fst $\mathrm{b} \longrightarrow(\mathrm{n} 1, \mathrm{n} 3) \notin$ snd $\mathrm{b})$; snd $\mathrm{n} 2=0 \mid] \Longrightarrow(\{\mathrm{n} 2\} \cup$ fst $\mathrm{b},\{(\mathrm{n} 1, \mathrm{n} 2)\} \cup$ snd $\mathrm{b}) \in$ bundles"

Our motivation here is two-folded. The more apparent is to introduce a more constructive definition to formalize a graph of a protocol session, rather that just saying that it is a casually well-defined graph. The other is to take advantage of the strong ability of induction principle supported by Isabelle to reason about inductively defined set. For the set of bundles, the induction principle says that $P(b)$ holds for each bundle $b$ provided that $P$ is preserved under all the rules for creating bundles.

\section{Path and Authentication Tests}

Definition 1. A path $p$ through bundle $b$ is a list of nodes such that $p=[]$, or $p_{0} \in b$ and $\left(p_{i}, p_{i+1}\right) \in(\rightarrow \cap$ snd $b)$ or $\left(p_{i}, p_{i+1}\right) \in(\Rightarrow \cap \text { snd } b)^{+}$for any $i$ such that $0 \leq i<$ length $p-1$.

If $a$ is uniquely originating from $n$, and $n^{\prime}$ is a node which contains $a$, and $n^{\prime}$ is in bundle $b$, then there exists a path $p$ through $b$ from $n$ to $n^{\prime}$, moreover, and if $p$ is across a positive node $m$ such that $a \sqsubset$ node_term $m$ and $m$ is in a non_originating strand for term $a$, then $p$ also crosses all nodes in $f$ st $m$ such that $m^{\prime} \Rightarrow^{+} m$ and $a \sqsubset$ node_term $m^{\prime}$; in particular, $p$ must cross a node $m^{\prime \prime}$, which is the first node containing $a$ in non-originating strand $f$ st $m$;

\section{Lemma 1}

$[\mid b \in$ bundles $\mid] \Longrightarrow$ uniquely_originate $a n \longrightarrow$ $\forall n^{\prime} . n^{\prime} \in$ fst $b \wedge a \sqsubset$ node_term $n^{\prime} \longrightarrow$

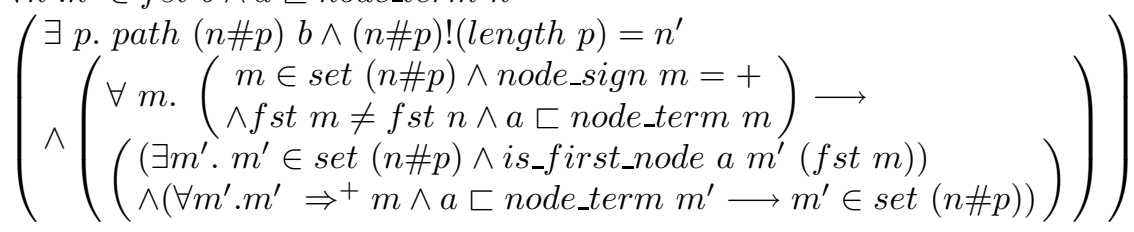


,where set $x s$ returns the set of all elements in list $x s$.

Proofs given by Guttman are extraordinarily complicated, we could not just follow his way to formalize results of authentication tests. Instead, we present authentication tests in a different style. Suppose that atom $a$ uniquely originates on $n$, and given a term set $T$ such that for all terms $t$ in $T$, if $a \sqsubset M$, then $t=$ Crypt $K M$, and invKey $K$ can not be obtained by the penetrator for some $K, M$; synth $T$ are terms that the penetrator can build up from $T$; if $n^{\prime}$ is another node containing $a$, and $n^{\prime}$ contains a new $a$-component which can not be built by synth $T$, furthermore, if we also assume that each component $t^{\prime}$ of each positive node on the originating strand is built by synth $T$, i.e., the originating strand does not help this transformation, then some regular strand except the originating one performs some transformation about $a$.

\section{Lemma 2 (outgoing-authentication-test).}

$[\mid b \in$ bundles; uniquely_originate a $n ; T \neq \varnothing ; a \in$ Atoms;

$\forall t \in T . \exists K M . a \sqsubset t \longrightarrow t=$ Crypt $K M$;

$\forall t . a \not \subset t \longrightarrow t \in T ; \forall k . a \neq K e y k ;$

$\forall t \in T . a \sqsubset t \longrightarrow \forall K M .\left(\begin{array}{l}t=\text { Crypt } K M \longrightarrow \\ \forall n .(\text { node_term } n=\text { invKey } K \rightarrow \text { attr } n \notin b a d)\end{array}\right)$;

$\forall m .\left(\begin{array}{l}f \text { st } m=f \text { st } n \wedge \text { node_sign } m=+\wedge a \sqsubset \text { node_term } m \longrightarrow \\ \text { node_term } m \in \text { synth } T\end{array}\right)$;

$a \sqsubset\left(\right.$ node_term $\left.m^{\prime}\right) \wedge$ node_term $m^{\prime} \notin$ synth $\left.T \mid\right] \Longrightarrow$

$\exists p m m^{\prime} .\left(\begin{array}{l}\text { path } n \# p b \wedge(n \# p) !(\text { length } p)=n^{\prime} \wedge \\ m \Rightarrow+m^{\prime} \wedge m \in \text { set }(n \# p) \wedge m^{\prime} \in \text { set }(n \# p) \wedge \\ \text { node_sign } m=-\wedge \text { node_sign } m^{\prime}=+\wedge \\ \text { attr }\left(\text { fst } m^{\prime}\right) \notin \text { bad } \wedge \text { st } m \neq \text { fst } n \\ \wedge \text { node_term } m \in \text { synth } T \\ \wedge \text { node_term } m^{\prime} \notin \text { synth } T \\ \wedge a \sqsubset \text { node_term } m \wedge a \sqsubset \text { node_term } m^{\prime}\end{array}\right)$

\section{Related Work and Conclusions}

Our work in this paper fruitfully borrows techniques from two popular approaches: strand space and the inductive method. Firstly, strand space model provides us a natural and efficient representation for the problem domain, and it has the advantage that it contains the exact casual relation information, and precise formulation about freshness assumption, and much more intuitive formulation of protocol's properties, and much simpler proofs. Our initial aim is just using Isabelle to formalise original strand space theory. But during this process, we find many concepts are too informal and many proofs needs a lot of human insight, and they are far away from being mechanized. In particular, when we try to formalise results of authentication tests, we find we could not overcome this difficulty if we do not extend their model. The definitions involved in results about normal form lemma and efficient form lemma are too tedious to formulate, and it is very hard to follow proofs even in paper proof, let alone to formalize them. Because all these problems are about bundles, they have given us the 
intuition that the crux lies in the definition of bundles, which is just a sketchy property specification, and not enough to support formal reasoning. But how to give a more tractable definition for bundles? Fortunately, Paulson's work in the inductive approach has inspired us to go ahead [3. Paulson's intuition behind his work has taught us that the principles of mathematical induction is not only simple, but also very powerful, especially to handle problems with infinite states. Besides, Isabelle's built-in support for inductive set and rule induction make it very convenient to apply induction principle. All these acted as stimuli for us to introduce the inductive approach to formalising strand space. The key to our approach is introducing an inductive definition for bundles, and it not only provides us a constructive illustration for a bundle, but also introduces an effective and rigorous technique of rule induction to prove properties of bundles. Using this induction principle, we can prove that a bundle is a casually wellfounded graph. We also clearly formalize the semantics to fresh assumption, and introduce the notion of complete transforming path and prove the existence of a $a$-complete transforming path through a bundle from its originating node to any nodes containing $a$. Combining the above results we give a rigorous proof for results of authentication tests. Our result of authentication test not only extends Guttman's result to a more general case, but also its proof is much easier and clearer, because we need not introduce normal bundles and efficient bundles. Our formulation and proof techniques are applicable generally. Analyzing a protocol case only requires definition about traces of regular strands, and our results of authentication tests make it very easy to prove authentication properties. As a trivial case study, we show how to use our results by proving authentication properties of Needham-Schroeder-Lowe protocol.

\section{References}

1. F. Javier Thayer Fabrega, Jonathan C. Herzog, Joshua D. Guttman. Strand Spaces: Proving Security Protocols Correct. Journal of Computer Security, 7 (1999), pages 191-230.

2. Guttman, J. D., Thayer Fabrega, F. J., 2000a. Authentication tests. In: Proceedings, 2000 IEEE Symposium on Security and Privacy. May, IEEE Computer Society Press.

3. L. C. Paulson. The Inductive Approach to Verifying Cryptographic Protocols. Journal of Computer Security, 6:85-128, 1998.

Appendix. Due to space limitation, we have to omit many details and references. Interesting readers can see http://lcs.ios.ac.cn/ ${ }^{\sim}$ lyj238/strand.html for them. 